ARTICLE

https://doi.org/10.1038/s41467-019-14248-1

\title{
A first principles method to determine speciation of carbonates in supercritical water
}

\author{
Ding Pan (i) ${ }^{1,2 \star} \&$ Giulia Galli $3,4,5$
}

The determination of the speciation of ions and molecules in supercritical aqueous fluids under pressure is critical to understanding their mass transport in the Earth's interior. Unfortunately, there is no experimental technique yet available to directly characterize species dissolved in water at extreme conditions. Here we present a strategy, based on firstprinciples simulations, to determine ratios of Raman scattering cross-sections of aqueous species under extreme conditions, thus providing a key quantity that can be used, in conjunction with Raman measurements, to predict chemical speciation in aqueous fluids. Due to the importance of the Earth's carbon cycle, we focus on carbonate and bicarbonate ions. Our calculations up to $11 \mathrm{GPa}$ and $1000 \mathrm{~K}$ indicate a higher concentration of bicarbonates in water than previously considered at conditions relevant to the Earth's upper mantle, with important implications for the transport of carbon in aqueous fluids in the Earth's interior.

\footnotetext{
${ }^{1}$ Department of Physics and Department of Chemistry, Hong Kong University of Science and Technology, Hong Kong, China. ${ }^{2}$ HKUST Fok Ying Tung Research Institute, Guangzhou, China. ${ }^{3}$ Pritzker School of Molecular Engineering, The University of Chicago, Chicago, IL 60637, USA. ${ }^{4}$ Department of Chemistry, The University of Chicago, Chicago, IL 60637, USA. ${ }^{5}$ Materials Science Division and Center for Molecular Engineering, Argonne National Laboratory, Argonne, IL 60439, USA. *email: dingpan@ust.hk
} 
T he carbon cycle in the atmosphere and biosphere has important consequences for the Earth's climate and thus is a topic of intense investigation in many fields of science, including chemistry, environmental science, and geoscience ${ }^{1,2}$. On the Earth, carbon is not only found in near-surface reservoirs, but it is also stored in the interior. In fact, $>90 \%$ of the Earth's carbon is buried in the Earth's interior ${ }^{3}$, and the carbon cycle in the deep Earth greatly influences the carbon budget near the surface, with a substantial impact on global climate change and human energy consumption ${ }^{4}$. After decades of study, our knowledge of the global carbon cycle in the atmosphere, oceans, and the shallow crust has improved considerably; however, relatively little is known about deep carbon reservoirs ${ }^{4,5}$. Water, an important component of geofluids in the Earth's crust and mantle, may carry a significant amount of carbon, but its dissolved forms in aqueous geofluids are still poorly known, thus limiting our overall understanding of mass transport of carbon in the Earth. This lack of knowledge is due to experimental difficulties in measuring which species are present in $\mathrm{C}-\mathrm{H}-\mathrm{O}$ fluids at extreme conditions, as well as to the use, on the theoretical side, of simplified models ${ }^{6,7}$. For example, $\mathrm{C}-\mathrm{H}-\mathrm{O}$ fluids at the conditions of the Earth's interior have often been modeled as mixtures of neutral gas molecules (e.g., refs. ${ }^{8,9}$ ). However, recent studies pointed out the importance of chemical reactions occurring in deep aqueous fluids, involving the presence of ionic products $^{10-12}$; for example, using first-principles simulations we recently suggested that the molecular $\mathrm{CO}_{2}(\mathrm{aq})$ species may completely convert into solvated carbonate and bicarbonate ions in water-rich fluids at high temperature and pressure ${ }^{13}$.

At present, the most used experimental tool to investigate chemical speciation in $\mathrm{C}-\mathrm{H}-\mathrm{O}$ fluids under extreme conditions is Raman spectroscopy ${ }^{14}$. The vibrational frequencies obtained from Raman spectra are used as fingerprints to identify molecules or ions, and in principle the concentration of chemical species may be obtained from vibrational peak intensities. Indeed, the concentration of a given species $\left(C_{i}\right)$ is proportional to the corresponding Raman intensity $I_{i}=\gamma_{i} C_{i}$, where $\gamma_{i}$ is the Raman scattering cross section. However, under extreme conditions, the variation of $\gamma_{i}$ with pressure $(\mathrm{P})$ and temperature $(\mathrm{T})$ is unknown; hence the concentration of chemical species may not be inferred from Raman peak intensities. Several estimates of the dependence of the Raman cross-sections of aqueous solutions at high P (HP) and high $\mathrm{T}(\mathrm{HT})$ have been reported in the literature; these estimates are not only controversial but in some cases they provide contradicting results. Based on geochemical models, Frantz predicted that the $\gamma$ ratio between carbonate and bicarbonate ions in aqueous fluids, $\frac{y_{\text {carb }}}{y_{\text {cito.t }}}$, decreased by $75 \%$ with increasing temperature from 523 to $823 \mathrm{~K}$, and did not vary with increasing pressure ${ }^{15}$. However, Schmidt suggested that this ratio changed by no $>10 \%$ from 296 to $873 \mathrm{~K}$ and up to $1 \mathrm{GPa}$, based on the comparison of the Raman bands of (bi)carbonate ions with those of similar ion pairs, sulfate and hydrogen sulfate ions, under similar conditions ${ }^{16}$.

The lack of knowledge of the $P-T$ dependence of Raman scattering cross-sections poses serious limitations to the use of Raman spectroscopy to determine chemical speciation of $\mathrm{C}-\mathrm{H}-\mathrm{O}$ fluids in the deep Earth, and as a consequence to our understanding of the deep carbon cycle, since at present Raman spectroscopy has been the technique of choice to investigate these liquids. Indeed the use of other spectroscopic methods, e.g., infrared ${ }^{17}$ or nuclear magnetic resonance ${ }^{18}$, is challenging under extreme $P-T$ conditions and it is not known how to derive the concentrations of dissolved species solely from measured spectroscopic data.

Here, we present a strategy, based on first-principles simulations at extreme conditions, to determine the ratios of the Raman scattering cross-sections, thus providing a key quantity that can

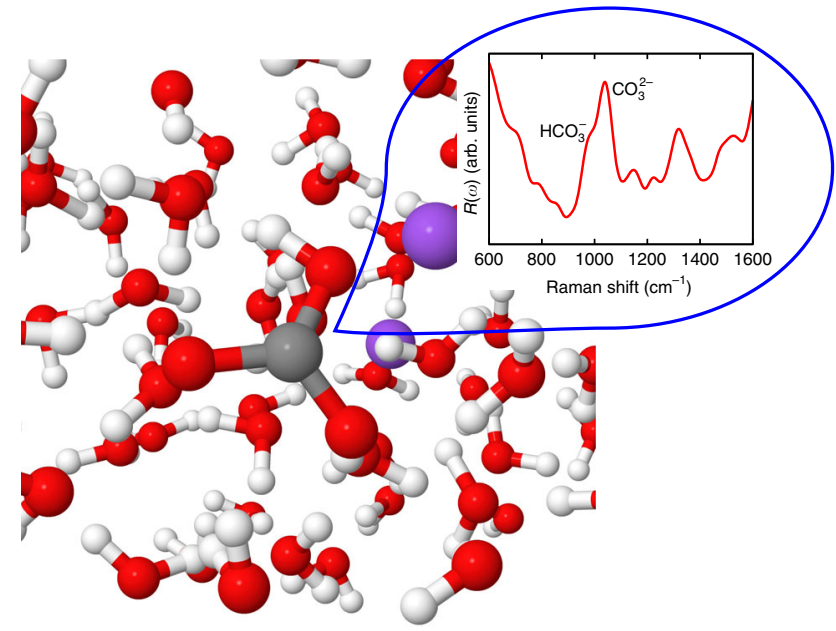

Fig. 1 A snapshot of the sodium carbonate solution at $\sim 10 \mathrm{GPa}$ and $1000 \mathrm{~K}$. The configuration was selected from first-principles molecular dynamics simulations trajectories (see text). The inset shows the calculated unpolarized Raman spectrum of the solution (red line).

be used, in conjunction with Raman measurements, to predict speciation in aqueous fluids. In particular we present the results of first-principles molecular dynamics (FPMD) simulations of $\mathrm{Na}_{2} \mathrm{CO}_{3}$ and $\mathrm{NaDCO}_{3}$ aqueous solutions at HP-HT conditions (see Fig. 1). Our simulations permit the determination of the concentration of species in the solution ${ }^{13}$, as well as of their Raman spectra, including peak positions and intensities. This combined information can then be used to obtain Raman crosssections. To the best of our knowledge, our study represents the first determination of Raman cross section ratios between aqueous carbonate and bicarbonate ions up to $\sim 11 \mathrm{GPa}$ and $1000 \mathrm{~K}$ and hence the first determination of speciation in these aqueous fluids. We find that this ratio decreases with increasing pressure, while it increases with increasing temperature, at variance from conclusions reached using geochemical models. Our results point at the presence of a higher concentration of bicarbonate ions at extreme conditions than previously thought, with important consequences for carbon transport in the Earth.

\section{Results}

Raman spectra at ambient density. Figure 2 shows the computed unpolarized and isotropic Raman spectra of $0.9 \mathrm{~mol} \mathrm{~L}^{-1} \mathrm{Na}_{2} \mathrm{CO}_{3}$ and $\mathrm{NaDCO}_{3}$ solutions at ambient density and $380 \mathrm{~K}$. We considered 380 instead of $300 \mathrm{~K}$ to better reproduce the experimental radial distribution functions and diffusion constant of water at ambient conditions with the functional adopted here $\left(\mathrm{PBE}^{19}\right)$. The solute concentration was set to a value similar to that determined experimentally ${ }^{15}$. The Raman spectra were obtained using FPMD simulations, which account for an-harmonic effects (see Methods), critical to describe fluids. At these conditions, we found that $<1 \%$ of carbonate and bicarbonate ions react in the $\mathrm{Na}_{2} \mathrm{CO}_{3}$ and $\mathrm{NaDCO}_{3}$ solutions. The broad band at $\sim 2400 \mathrm{~cm}^{-1}$ shown in the figure originates from the water stretching mode ${ }^{20}$. The unpolarized and isotropic spectra show similar features, except at low frequency, in the range of $0 \sim 700 \mathrm{~cm}^{-1}$, where the baselines of the isotropic spectra are closer to zero than those of the unpolarized ones, consistent with experimental observations $^{21}$. Following a procedure commonly adopted in experiments ${ }^{21}$, we used isotropic spectra to obtain Raman peak intensities. We first analyze the weak peaks between 400 and $1800 \mathrm{~cm}^{-1}$ (see Fig. 3). We assigned those peaks by using the 


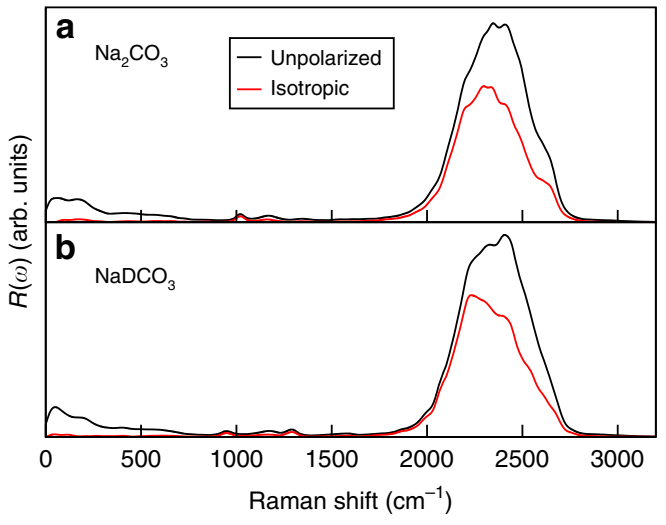

Fig. 2 Raman spectra of $0.9 \mathrm{~mol} \mathrm{~L}^{-1}$ solutions at ambient density and $380 \mathrm{~K}$. Calculated unpolarized (black line) and isotropic (red line) spectra of the sodium carbonate and deuterated sodium bicarbonate solutions are shown in the upper and lower panels, respectively.

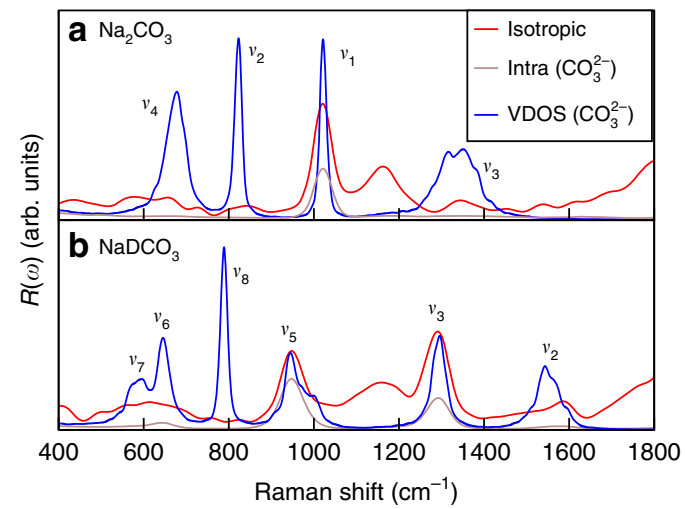

Fig. 3 Zoomed-in Raman spectra of $0.9 \mathrm{~mol} \mathrm{~L}^{-1}$ solutions at ambient density and $380 \mathrm{~K}$. Calculated spectra of the sodium carbonate and deuterated sodium bicarbonate solutions are shown in the upper and lower panels, respectively. The isotropic Raman spectra (red line), the intramolecular Raman spectra (brown line), and the vibrational density of states (VDOS) (blue line) of $\mathrm{CO}_{3}^{2-}$ are compared.

computed vibrational densities of states (VDOS) and the intramolecular Raman spectrum of the $\mathrm{CO}_{3}^{2-}$ species. The VDOS of $\mathrm{CO}_{3}^{2-}$ was obtained by Fourier transforming the velocity-velocity auto-correlation function:

$$
I_{\mathrm{CO}_{3}^{2-}}^{\mathrm{VDOS}}=\int d t \mathrm{e}^{-\mathrm{i} \omega t} \frac{\sum_{i \in \mathrm{CO}_{3}^{2-}}\left\langle\mathbf{v}_{i}(0) \cdot \mathbf{v}_{i}(t)\right\rangle}{\sum_{i \in \mathrm{CO}_{3}^{2-}}\left\langle\mathbf{v}_{i}(0) \cdot \mathbf{v}_{i}(0)\right\rangle},
$$

where $t$ is the correlation time, $\omega$ is the frequency, and $\mathbf{v}_{i}$ is the velocity of the $i$ th atom in the $\mathrm{CO}_{3}^{2-}$ unit. The intramolecular Raman spectra of $\mathrm{CO}_{3}^{2-}$ were computed using the auto-correlation functions of the effective polarizabilities of $\mathrm{CO}_{3}^{2-}, \alpha_{\mathrm{CO}_{3}^{2-}}$ :

$$
\mu_{\mathrm{CO}_{3}^{2-}}=\alpha_{\mathrm{CO}_{3}^{2-}} \mathbf{E}
$$

In Eq. (2), $\mu_{\mathrm{CO}_{3}^{2-}}$ is the induced polarization of $\mathrm{CO}_{3}^{2-}$ (computed from the centers of maximally localized Wannier functions) and E is the macroscopic electric field (see Supplementary Methods).

The carbonate ion has four normal vibrational modes ${ }^{22}$, clearly visible in the VDOS of $\mathrm{CO}_{3}^{2-}$ : the $v_{1}$ symmetric stretching at $1021 \mathrm{~cm}^{-1}$, the $v_{2}$ out-of-plane deformation at $823 \mathrm{~cm}^{-1}$, the $v_{3}$ asymmetric stretching at $\sim 1350 \mathrm{~cm}^{-1}$, and the the $v_{4}$ in-plane deformation at $678 \mathrm{~cm}^{-1}$. In the full and intra-molecular Raman spectra, the $v_{1}$ peak is the most intense, and it has thus been used

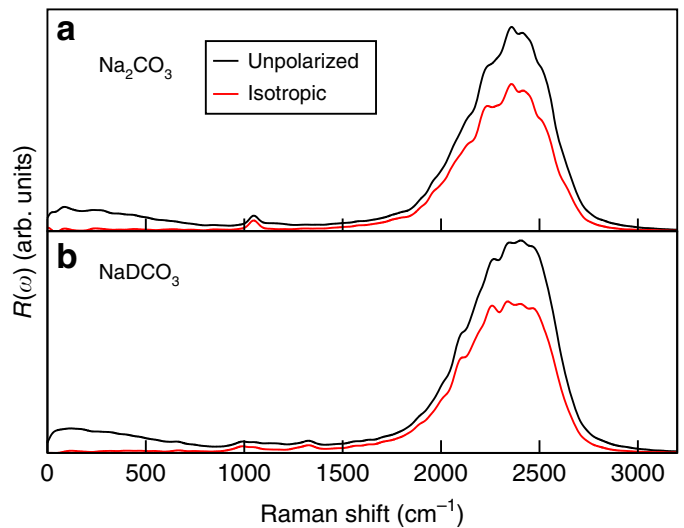

Fig. 4 Raman spectra of 0.9 molality solutions at $\sim 9 \mathrm{GPa}$ and $600 \mathrm{~K}$. Calculated unpolarized (black line) and isotropic (red line) spectra of the sodium carbonate and deuterated sodium bicarbonate solutions are shown in the upper and lower panels, respectively.

as the characteristic Raman signature of carbonate ions. The bicarbonate ion has 9 normal vibrational modes ${ }^{22}$. In Fig. 3, the VDOS corresponding to the bicarbonate ion $\mathrm{HCO}_{3}^{-}$show at least six vibrational modes: the $v_{7}(\mathrm{OD})$ and $v_{6}(\mathrm{CO})$ bendings at $\sim 596 \mathrm{~cm}^{-1}$ and $645 \mathrm{~cm}^{-1}$, respectively, the $v_{8}\left(\mathrm{CO}_{3}\right)$ out-of-plane deformation at $788 \mathrm{~cm}^{-1}$, the $v_{5}(\mathrm{C}-\mathrm{OD})$ symmetric stretching at $945 \mathrm{~cm}^{-1}$, the $v_{3}(\mathrm{CO})$ symmetric stretching at $1296 \mathrm{~cm}^{-1}$, and the $v_{2}(\mathrm{CO})$ asymmetric stretching at $\sim 1542 \mathrm{~cm}^{-1}$. In the full and intra-molecular Raman spectra, only the $v_{5}(\mathrm{C}-\mathrm{OD})$ and $v_{3}(\mathrm{CO})$ modes can be easily identified. In both Raman spectra of the carbonate and bicarbonate solutions we found a broad band at $\sim 1150 \mathrm{~cm}^{-1}$, which is not present in the VDOS and intramolecular Raman spectra of $\mathrm{CO}_{3}^{2-}$. A similar band can be found in the Raman spectrum of pure water at ambient conditions ${ }^{20}$, and hence it was assigned to the bending mode of liquid water. We note that the calculated Raman frequencies are systematically lower than the experimental ones by $\sim 40 \mathrm{~cm}^{-1}$ (ref. ${ }^{23}$ ); this underestimate is ascribed to the exchange-correlation functional chosen in our simulations (a gradient-corrected functional: $\left.\mathrm{PBE}^{19}\right)$. It is well known that generalized gradient approximations underestimate the vibrational frequencies of covalent bonds ${ }^{24}$ and we do not consider this underestimate a serious drawback of our simulations, which we are using to assign species and understand trends, rather than to obtain exact values of vibrational frequencies. Importantly, our calculated unpolarized and isotropic Raman spectra are overall in good agreement with experimental ones. We also note that quite a few modes are present in the VDOS but not in the computed Raman spectra, indicating that the Raman selection rules included in our calculations are correctly reproducing experimental findings.

Raman spectra at high pressure and temperature. Having interpreted the low pressure and temperature spectra, we turned to solutions under extreme conditions. We increased the pressure of our simulations to $\sim 9 \mathrm{GPa}$ and the temperature to $600 \mathrm{~K}$. At these conditions we observed the conversion between carbonate and bicarbonate ions. In the molecular dynamics (MD) simulation of $0.9 \mathrm{~m}$ (molality) $\mathrm{Na}_{2} \mathrm{CO}_{3}$, the mole percent of $\mathrm{DCO}_{3}^{-}$per total carbon species is $8.5 \%$ and the remaining carbon species are $\mathrm{CO}_{3}^{2-}$. In the $0.9 \mathrm{~m} \mathrm{NaDCO}_{3}$ solution, we found instead $18.5 \%$ $\mathrm{CO}_{3}^{2-}, 81.3 \% \mathrm{DCO}_{3}^{-}$, and $0.2 \% \mathrm{D}_{2} \mathrm{CO}_{3}$. The mole percents were obtained from MD trajectories based on the molecular geometry of the solutes (see the Methods section in ref. ${ }^{13}$ ). Figure 4 shows the full Raman spectra of these two solutions, and Fig. 5 shows a specific frequency region. The calculated spectra are overall 


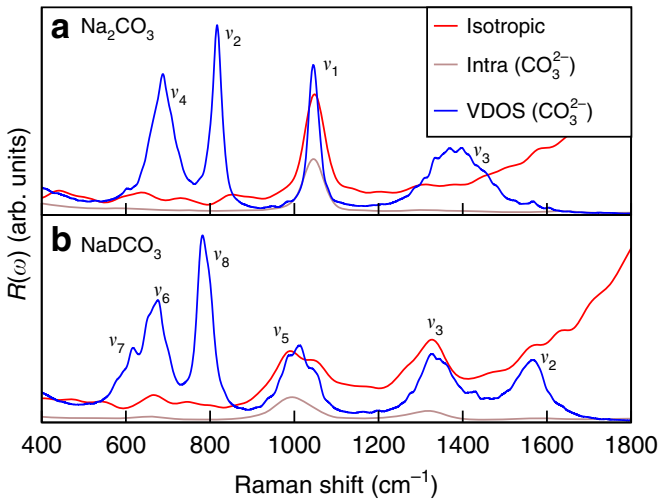

Fig. 5 Zoomed-in Raman spectra of 0.9 molality solutions at $\sim 9 \mathrm{GPa}$ and $600 \mathrm{~K}$. Calculated spectra of the sodium carbonate and deuterated sodium bicarbonate solutions are shown in the upper and lower panels, respectively. The isotropic Raman spectra (red line), the intra-molecular Raman spectra (brown line), and the vibrational density of states (VDOS) (blue line) of $\mathrm{CO}_{3}^{2-}$ are compared.

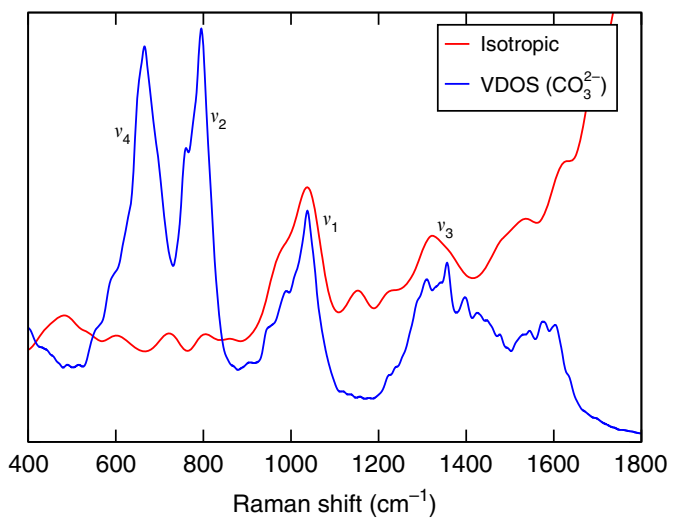

Fig. 6 Raman spectra of the $\mathbf{0 . 9}$ molality sodium carbonate solution at $\sim 11 \mathrm{GPa}$ and $1000 \mathrm{~K}$. The isotropic Raman spectrum of this solution (red line) and the vibrational density of states (VDOS) (blue line) of $\mathrm{CO}_{3}^{2-}$ are compared.

similar to those obtained at ambient conditions, but the vibrational bands are wider and the Raman active modes, $v_{1}$ of carbonate ions, $v_{5}(\mathrm{C}-\mathrm{OD})$ and $v_{3}(\mathrm{CO})$ of bicarbonate ions, are all shifted to higher frequencies relative to ambient conditions. It has been experimentally reported that the Raman frequencies of the $v_{1}$ and $v_{5}(\mathrm{C}-\mathrm{OH})$ modes both increase with increasing pressure at a rate of $\sim 6.5$ and $\sim 14 \mathrm{~cm}^{-1}$ per GPa, respectively, and decrease with increasing temperature at a rate of $\sim-0.035$ and $\sim-0.096 \mathrm{~cm}^{-1}$ per K, respectively ${ }^{16}$; hence our results, obtained at $\sim 9 \mathrm{GPa}$ and $600 \mathrm{~K}$, indicate that the pressure effect outweighs the temperature one and all major Raman peaks are blue-shifted compared to those at ambient conditions.

Upon further increase of the pressure of the $0.9 \mathrm{~m} \mathrm{Na}_{2} \mathrm{CO}_{3}$ solution to $\sim 11 \mathrm{GPa}$ and the temperature to $1000 \mathrm{~K}$, we found $40.6 \% \quad \mathrm{CO}_{3}^{2-}, \quad 57.7 \% \quad \mathrm{DCO}_{3}^{-}$, and $1.6 \% \mathrm{D}_{2} \mathrm{CO}_{3}$. At these conditions, the carbonate and bicarbonate ions are rapidly converting into each other, and their lifetime is estimated to be on the sub-picosecond time scale, as reported in our previous study ${ }^{13}$. Figure 6 shows the isotropic Raman spectrum and VDOS of this solution. The Raman peaks are much broadened, due to the decreased lifetime of ions in the solution. Again, with the help of the computed VDOS and the intra-molecular Raman spectrum of $\mathrm{CO}_{3}^{2-}$, we identified the $v_{1}$ mode of carbonate ions and the $v_{3}(\mathrm{CO})$ mode of bicarbonate ions. The $v_{1}$ band exhibits a shoulder, which is primarily assigned to the $v_{5}(\mathrm{C}-\mathrm{OD})$ mode of bicarbonate ions. The Raman signal of ion pairs $\mathrm{NaCO}_{3}^{-}$is expected to be between the $v_{1}$ and $v_{5}(\mathrm{C}-\mathrm{OD}) \mathrm{bands}^{16}$.

We note that while our calculations for the bicarbonate ion show the presence of two Raman bands (see Fig. 5), $v_{5}(\mathrm{C}-\mathrm{OD})$ and $v_{3}(\mathrm{CO})$, in high-pressure experiments conducted in diamond anvil cells, only the $v_{5}(\mathrm{C}$-OD) mode is detected. The reason is that the Raman band of the diamond window is at $1332 \mathrm{~cm}^{-1}$, i.e., very close to the Raman frequency of the $v_{3}(\mathrm{CO})$ mode, and it becomes broader at high $P$ and $T^{25}$ and thus almost indistinguishable from the $v_{3}(\mathrm{CO})$ mode.

\section{Discussion}

Having assigned all peaks in our computed spectra, and having determined the corresponding concentrations of ionic species from the MD trajectories, we are now in a position to directly compute $\gamma$ from the Raman peak intensities and the concentrations of species. As mentioned in the introduction, $\gamma$ is precisely the information not directly available in experiments at high $P$ and $T$, and required to determine speciation from measured Raman spectra. We recall that at ambient conditions, and at 600 $\mathrm{K}$ and $\sim 9 \mathrm{GPa}$, we calculated the Raman spectra of $\mathrm{Na}_{2} \mathrm{CO}_{3}$ and $\mathrm{NaDCO}_{3}$ solutions in separate simulations; therefore before proceeding to the determination of the $\gamma$ ratio, the two spectra obtained in independent simulations need to be aligned. Since we simulated the $\mathrm{Na}_{2} \mathrm{CO}_{3}$ and $\mathrm{NaDCO}_{3}$ solutions with the same number of water molecules, we can use the water stretching band as our calibration to align the spectra. We found that the calculated $\gamma$ ratio decreases with increasing Gaussian broadening width used to smooth the computed spectra; hence for comparison purposes, we adopted the same width in all the Raman spectra. The $\gamma$-ratio, $\frac{\gamma_{\text {carb }}}{\gamma_{\text {biart }}}$, obtained from our simulations at ambient conditions is 1.57 , which is comparable to the experimental value 1.46 reported by Rudolph et al. ${ }^{21}$, who also observed that the $\gamma$-ratio did not change up to $492 \mathrm{~K}$. Both the experimental and calculated $\gamma$-ratios suggest that the Raman scattering cross section of carbonate ions is larger than that of bicarbonate ions, consistent with the expectation that more symmetric species, such as the carbonate ion, have a larger $\gamma$ for the symmetric stretching modes. At $600 \mathrm{~K}$ and $\sim 9 \mathrm{GPa}$, the computed $\gamma$ ratio is 1.60 , essentially the same as that found at ambient conditions. At $1000 \mathrm{~K}$ and $\sim 11 \mathrm{GPa}$, the determination of the $\gamma$ ratio is much more challenging, because the Raman signal of bicarbonate ions is hidden in the shoulder of the Raman peak of carbonate ions at $1037 \mathrm{~cm}^{-1}$. In this case no peak alignment was needed, because both ions were simulated in the same solution. We found a weak peak at $989 \mathrm{~cm}^{-1}$ in the VDOS of $\mathrm{CO}_{3}^{2-}$, which may be assigned to the $v_{5}(\mathrm{C}-\mathrm{OD})$ mode of bicarbonate ions. Using the Raman intensities at $989 \mathrm{~cm}^{-1}$ and $1037 \mathrm{~cm}^{-1}$, we calculated the $\gamma$ ratio to be 1.81 , which is larger than that at ambient conditions, and at $600 \mathrm{~K}$ and $\sim 9 \mathrm{GPa}$.

To understand how pressure and temperature separately affect the value of $\gamma$, we computed the Raman spectra of the $\delta-\mathrm{Na}_{2} \mathrm{CO}_{3}$ and $\mathrm{NaHCO}_{3}$ crystals up to $10 \mathrm{GPa}$ and at $0 \mathrm{~K}$ in Fig. 7. The space groups of these two crystals are $C 2 / \mathrm{m}^{26}$ and $P 12_{\gamma} / c 1^{27}$, respectively. We compressed the crystals without allowing for structural phase transitions. Figure 7 shows that both $\gamma_{\text {carb }}$ and $\gamma_{\text {bicarb }}$ increase with increasing pressure, but $\gamma_{\text {bicarb }}$ increases faster than $\gamma_{\text {carb }}$, so the $\gamma$ ratio decreases by $17 \%$ from 0 to $10 \mathrm{GPa}$. Our FPMD simulations showed that the $\gamma$ ratio is approximately constant in going from ambient conditions to $\sim 9 \mathrm{GPa}$ and $600 \mathrm{~K}$; this result, together with the one obtained for the solids under pressure, indicates that the $\gamma$ ratio increases with increasing temperature. If we assume that this ratio linearly depends separately on temperature and pressure, then its value may be 


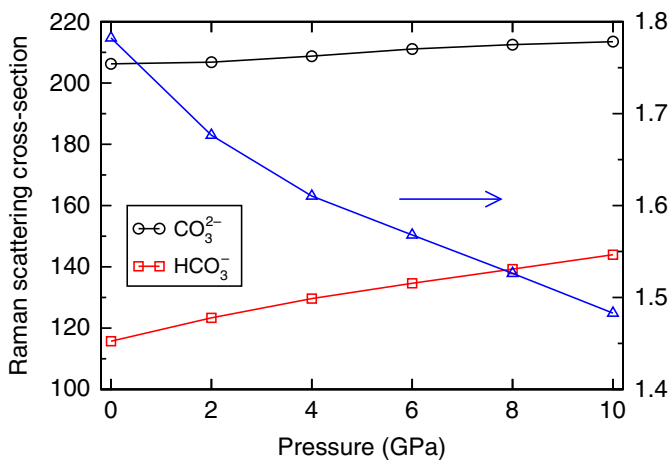

Fig. 7 Raman scattering cross-sections of carbonate and bicarbonate

ions. We present results for the $\delta-\mathrm{Na}_{2} \mathrm{CO}_{3}$ (black line) and $\mathrm{NaHCO}_{3}$ (red line) crystals as a function of pressure. The cross section ratios between these two ions, i.e., the $\gamma$ ratio $\left(\frac{\gamma_{\text {cart }}}{\gamma_{\text {biarb }}}\right)$, are shown as blue triangles.

obtained from the following relation:

$$
\frac{\gamma_{\text {carb }}}{\gamma_{\text {bicarb }}}(P, T)=\frac{\gamma_{\text {carb }}}{\gamma_{\text {bicarb }}}\left(P_{0}, T_{0}\right)+\alpha\left(P-P_{0}\right)+\beta\left(T-T_{0}\right),
$$

where $\alpha$ is the decrease rate with pressure and $\beta$ is the increase rate with temperature. Using the $\gamma$ ratios in Fig. 7 and at $\sim 9 \mathrm{GPa}$ and $600 \mathrm{~K}$, we can calculate $\alpha$ as $-2.99 \times 10^{-2} \mathrm{GPa}^{-1}$ and $\beta$ as $9.84 \times 10^{-4} \mathrm{~K}^{-1}$. To test the validity of Eq. (3), we used the $\alpha$ - and $\beta$-values obtained above to calculate the $\gamma$ ratio at $\sim 11 \mathrm{GPa}$ and $1000 \mathrm{~K}$, which we also have computed directly from FP simulations: we obtained a value of 1.93 , about $7 \%$ larger than the one directly obtained from the Raman spectrum in Fig. 6 (1.81). This agreement is not perfect but satisfactory and importantly we found the same trend relative to ambient conditions from our simulations and using Eq. (3).

Our calculation shows that the $\gamma$-ratio increases with increasing temperature, but decreases with increasing pressure; these results differ from those obtained from geochemical models. Using the concentrations calculated from geochemical models, Frantz predicted that the $\gamma$-ratio decreased by $75 \%$ with increasing temperature from 523 to $823 \mathrm{~K}$, and did not change with increasing pressure ${ }^{15}$. However, Schmidt reported that the $\gamma$-ratio estimated at ambient conditions from Frantz's work (0.98) is much smaller than the experimental value (1.46), casting doubts on the generality of Frantz's model ${ }^{16}$. In addition, at $873 \mathrm{~K}$ the $\gamma$-ratio given by Frantz is only 0.003 , indicating that the Raman bands of $\mathrm{CO}_{3}^{2-}$ are extremely weak and almost impossible to detect, contrary to what observed experimentally ${ }^{16}$. Hence, it is likely that the carbon species concentrations given by the geochemical models in Frantz's work are not accurate. According to Rudolph's Raman studies, the $\gamma$ ratio changes little from 296 to $492 \mathrm{~K}^{21}$. Furthermore, Schmidt suspected that this ratio should not change $>10 \%$ from 492 to $873 \mathrm{~K}$ and up to $1 \mathrm{GPa}$, but according to Eq. (3), this ratio should increase by $22 \%$ in this $P-T$ range. At high temperatures, underestimating the $\gamma$ ratio leads to an underestimate of the concentration of bicarbonate ions. In our previous study of the $\mathrm{Na}_{2} \mathrm{CO}_{3}$ solutions at $0.2 \mathrm{GPa}$ and $823 \mathrm{~K}$, the mole percent of $\mathrm{HCO}_{3}^{-}$obtained by Raman spectra and the $\gamma$ ratio at ambient conditions is $68 \%$, which is smaller than the mole percent obtained by the FPMD simulations (73-82\%), showing that the concentration of bicarbonate ions is indeed underestimated if we assume that the $\gamma$ ratio does not change with pressure and temperature.

In summary, we presented a strategy to determine the speciation of carbonates in aqueous fluids under pressure, which combines theoretical and experimental spectroscopies. We considered conditions relevant to the Earth's upper mantle, though our first-principles calculations can be conducted under a variety of different conditions and for numerous other ions and molecules in aqueous environments. In particular we showed how to compute, from first-principles, Raman scattering cross-sections, which are not yet known experimentally, at high pressure and high temperature. The information on cross-sections, together with that of measured Raman intensities, can then be used to obtain the speciation of aqueous carbonates, an essential prerequisite to understand the chemistry of carbon in water at extreme conditions.

Interestingly, we found that the ratio of the Raman scattering cross-sections of carbonate and bicarbonate ions in water, $\frac{\gamma_{\text {carb }}}{\gamma_{\text {biart }}}$, decreases with increasing pressure, while it increases with increasing temperature, up to $\sim 10 \mathrm{GPa}$ and $1000 \mathrm{~K}$. Our results differ from those reported previously based on geochemical models, showing either a decrease or a constant $\gamma$ ratio under high temperature. These discrepancies are not surprising as the $\mathrm{C}-\mathrm{H}-\mathrm{O}$ fluids were modeled assuming neutral gas species under extreme $P-T$ conditions, while many recent studies, including our simulations, show reactions between these gas species in aqueous environments. The results obtained here for the $\gamma$ ratio imply a higher concentration of bicarbonate ions than previously considered, with important implications for the transport of carbon in aqueous fluids in the Earth interior.

\section{Methods}

First-principles molecular dynamics. We performed first-principles MD simulations using the Qbox code (http://qboxcode.org) ${ }^{28}$. The time step was $0.24 \mathrm{fs}$. We used the PBE exchange-correlation functional ${ }^{19}$ and Hamann-SchluterChiang-Vanderbilt norm-conserving pseudopotentials ${ }^{29,30}$ with a plane-wave kinetic energy cutoff of $85 \mathrm{Ry}$. When calculating pressure, the cutoff was increased to $220 \mathrm{Ry}$. There are 62 water molecules and one $\mathrm{Na}_{2} \mathrm{CO}_{3}$ or $\mathrm{NaDCO}_{3}$ molecule in the cubic simulation box with periodic boundary conditions. We used deuterium instead of hydrogen so as to enable the use of a larger time step in MD simulations Temperature was controlled by the Bussi-Donadio-Parrinello thermostat ${ }^{31}$, and the time scale of the response of the thermostat $(\tau)$ is $24.2 \mathrm{fs}$.

First-principles Raman spectroscopy. The Raman spectra were calculated by the auto-correlation functions of electronic polarizabilities ${ }^{20}$. The isotropic and anisotropic Raman spectra were obtained respectively by

$$
\begin{gathered}
R_{\text {iso }}(\omega) \propto \frac{\hbar \omega}{k_{B} T} \int d t \mathrm{e}^{-\mathrm{i} \omega t}\langle\bar{\alpha}(0) \bar{\alpha}(t)\rangle \\
R_{\text {aniso }}(\omega) \propto \frac{\hbar \omega}{k_{B} T} \int d t \mathrm{e}^{-\mathrm{i} \omega t}\left\langle\frac{2}{15} \operatorname{Tr} \beta(0) \beta(t)\right\rangle,
\end{gathered}
$$

where $\omega$ is the frequency, $k_{B}$ is the Boltzmann constant, $t$ is the correlation time, and $\operatorname{Tr}$ is the trace matrix operator, $\bar{\alpha}$ and $\beta$ are the isotropic and anisotropic components of the polarizability tensor $\alpha: \bar{\alpha}=\frac{1}{3} \operatorname{Tr} \alpha$ and $\beta=\alpha-\bar{\alpha} \mathbf{I}$, where $\mathbf{I}$ is the identity tensor. The linear combination of these two spectra gives the unpolarized Raman spectra: $R_{\text {unpol }}=R_{\text {iso }}+\frac{7}{4} R_{\text {aniso }}$. The polarizability tensors were calculated by density functional perturbation theory ${ }^{32}$ every $15 \mathrm{MD}$ steps. The length of each MD trajectory used in the auto-correlation calculations is between 210 and $280 \mathrm{ps}$ A Gaussian broadening is used to smooth spectra, whose full width at half maximum (FWHM) is $45 \mathrm{~cm}^{-1}$.

We note that in our MD simulations, nuclei were treated as classical particles, the comparisons between intensities reported for the vibrational spectra of water with classical and quantum dynamics at ambient conditions do not show any qualitative difference and relatively small quantitative differences for the intensities, with blue-shifted frequencies of the order $60-120 \mathrm{~cm}^{-1}$ in the case of classical dynamics $^{33,34}$. At elevated temperatures, we expect the small differences observed at ambient conditions to diminish.

\section{Data availability}

The data that support this study are available upon request from the authors.

\section{Code availability}

Qbox is a free and open source code available at http://qboxcode.org. Data processing scripts are available upon request from the authors.

Received: 4 July 2019; Accepted: 4 December 2019; Published online: 21 January 2020 


\section{References}

1. Falkowski, P. et al. The global carbon cycle: a test of our knowledge of earth as a system. Science 290, 291-296 (2000).

2. Hayes, J. M. \& Waldbauer, J. R. The carbon cycle and associated redox processes through time. Philos. Trans. Royal Soc. B 361, 931-950 (2006).

3. Javoy, M. The major volatile elements of the Earth: Their origin, behavior, and fate. Geophys. Res. Lett. 24, 177-180 (1997).

4. Hazen, R. M. \& Schiffries, C. M. Why deep carbon? Rev. Mineral. Geochem. 75, 1-6 (2013)

5. Manning, C. E. Geochemistry: a piece of the deep carbon puzzle. Nature Geosci. 7, 333-334 (2014).

6. Manning, C. E., Shock, E. L. \& Sverjensky, D. The chemistry of carbon in aqueous fluids at crustal and upper mantle conditions: experimental and theoretical constraints. Rev. Mineral Geochem. 75, 109-148 (2013).

7. Manning, C. E. Fluids of the lower crust: Deep is different. Annu. Rev. Earth Planet. Sci. 46, 67-97 (2018).

8. Huizenga, J.-M. Thermodynamic modelling of $\mathrm{C}-\mathrm{O}-\mathrm{H}$ fluids. Lithos 55, 101-114 (2001).

9. Zhang, C. \& Duan, Z. A model for COH fluid in the earth's mantle. Geochim. Cosmochim. Acta 73, 2089-2102 (2009).

10. Facq, S., Daniel, I., Montagnac, G., Cardon, H. \& Sverjensky, D. A. In situ raman study and thermodynamic model of aqueous carbonate speciation in equilibrium with aragonite under subduction zone conditions. Geochim. Cosmochim. Acta 132, 375-390 (2014).

11. Abramson, E. H., Bollengier, O. \& Brown, J. M. The water-carbon dioxide miscibility surface to $450^{\circ} \mathrm{C}$ and $7 \mathrm{GPa}$. Am. J. Sci. 317, 967-989 (2017).

12. Sverjensky, D. A. Thermodynamic modelling of fluids from surficial to mantle conditions. J. Geol. Soc. 176, 348-374 (2019).

13. Pan, D. \& Galli, G. The fate of carbon dioxide in water-rich fluids under extreme conditions. Sci. Adv. 2, e1601278 (2016)

14. Goncharov, A. F. Raman spectroscopy at high pressures. Int. J. Spectrosc. 2012, 617528 (2012).

15. Frantz, J. D. Raman spectra of potassium carbonate and bicarbonate aqueous fluids at elevated temperatures and pressures: comparison with theoretical simulations. Chem. Geol. 152, 211-225 (1998).

16. Schmidt, C. Raman spectroscopic determination of carbon speciation and quartz solubility in $\mathrm{H}_{2} \mathrm{O}+\mathrm{Na}_{2} \mathrm{CO}_{3}$ and $\mathrm{H}_{2} \mathrm{O}+\mathrm{NaHCO}_{3}$ fluids to $600{ }^{\circ} \mathrm{C}$ and 1.53 GPa. Geochim. Cosmochim. Acta 145, 281-296 (2014).

17. Sowerby, J. R. \& Keppler, H. Water speciation in rhyolitic melt determined by in-situ infrared spectroscopy. Am. Mineral. 84, 1843-1849 (1999).

18. Pilgrim, C. D., Casey, W. H. \& Walton, J. H. High pressure aqueous geochemical NMR. eMagRes 8, 127-136 (2019).

19. Perdew, J. P., Burke, K. \& Ernzerhof, M. Generalized gradient approximation made simple. Phys. Rev. Lett. 77, 3865 (1996).

20. Wan, Q., Spanu, L., Galli, G. A. \& Gygi, F. Raman spectra of liquid water from ab initio molecular dynamics: vibrational signatures of charge fluctuations in the hydrogen bond network. J. Chem. Theory Comput. 9, 4124-4130 (2013).

21. Rudolph, W. W., Irmer, G. and Königsberger, E. Speciation studies in aqueous $\mathrm{HCO}_{3}^{-}-\mathrm{CO}_{3}^{2-}$ solutions. A combined raman spectroscopic and thermodynamic study. Dalton Trans. 900-908, https://doi.org/10.1039/ B713254A (2008).

22. Davis, A. \& Oliver, B. A vibrational-spectroscopic study of the species present in the $\mathrm{CO}_{2}-\mathrm{H}_{2} \mathrm{O}$ system. J. Solution Chem. 1, 329-339 (1972)

23. Rudolph, W. W., Fischer, D. \& Irmer, G. Vibrational spectroscopic studies and density functional theory calculations of speciation in the $\mathrm{CO}_{2}$-Water system. Appl. Spectrosc. 60, 130-144 (2006).

24. Boulard, E., Pan, D., Galli, G., Liu, Z. \& Mao, W. L. Tetrahedrally coordinated carbonates in earth's lower mantle. Nat. Commun. 6, 6311 (2015).

25. Goncharov, A. F. et al. Dynamic ionization of water under extreme conditions. Phys. Rev. Lett. 94, 125508 (2005).

26. Dušek, M., Chapuis, G., Meyer, M. \& Petricek, V. Sodium carbonate revisited. Acta Crystallogr. B 59, 337-352 (2003).

27. Duan, Y. et al. Density functional theory studies on the electronic, structural, phonon dynamical and thermo-stability properties of bicarbonates $\mathrm{MHCO}_{3}$, $\mathrm{M}=\mathrm{Li}, \mathrm{Na}, \mathrm{K}$. J. Phys. Condens. Matter 24, 325501 (2012).

28. Gygi, F. Architecture of qbox: A scalable first-principles molecular dynamics code. IBM J. Res. Dev. 52, 137-144 (2008).
29. Hamann, D. R., Schlüter, M. \& Chiang, C. Norm-conserving pseudopotentials. Phys. Rev. Lett. 43, 1494-1497 (1979).

30. Vanderbilt, D. Optimally smooth norm-conserving pseudopotentials. Phys Rev. B 32, 8412-8415 (1985).

31. Bussi, G., Donadio, D. \& Parrinello, M. Canonical sampling through velocity rescaling. J. Chem. Phys. 126, 014101 (2007).

32. Baroni, S., De Gironcoli, S., Dal Corso, A. \& Giannozzi, P. Phonons and related crystal properties from density-functional perturbation theory. Rev. Mod. Phys. 73, 515 (2001).

33. Reddy, S. K., Moberg, D. R., Straight, S. C. \& Paesani, F. Temperaturedependent vibrational spectra and structure of liquid water from classical and quantum simulations with the mb-pol potential energy function. J. Chem. Phys. 147, 244504 (2017).

34. Morawietz, T. et al. The interplay of structure and dynamics in the raman spectrum of liquid water over the full frequency and temperature range. $J$. Phys. Chem. Lett. 9, 851-857 (2018).

\section{Acknowledgements}

We thank Quan Wan for helpful discussions and Shenli Zhang for her help in the final revision of our manuscript. D.P. acknowledges support from Hong Kong Research

Grants Council (project number ECS-26305017, GRF-16307618), the National Natural Science Foundation of China (project number 11774072), the Alfred P. Sloan Foundation through the Deep Carbon Observatory (DCO), and the Croucher Foundation through the Croucher Innovation Award. Work by Giulia Galli was supported by AMEWS, an Energy Frontier Research Center funded by DOE, Office of Science, Basic Energy Science. Part of this work was carried out using the DCO Computer Cluster. We also acknowledge the University of Chicago Research Computing Center for support of this work.

\section{Author contributions}

D.P. and G.G. designed the research. Calculations were performed by D.P. All authors contributed to the analysis and discussion of the data and the writing of the manuscript.

\section{Competing interests}

The authors declare no competing interests.

\section{Additional information}

Supplementary information is available for this paper at https://doi.org/10.1038/s41467 019-14248-1.

Correspondence and requests for materials should be addressed to D.P.

Peer review information Nature Communications thanks the anonymous reviewer(s) for their contribution to the peer review of this work.

Reprints and permission information is available at http://www.nature.com/reprints

Publisher's note Springer Nature remains neutral with regard to jurisdictional claims in published maps and institutional affiliations.

Open Access This article is licensed under a Creative Common Attribution 4.0 International License, which permits use, sharing, adaptation, distribution and reproduction in any medium or format, as long as you give appropriate credit to the original author(s) and the source, provide a link to the Creative Commons license, and indicate if changes were made. The images or other third party material in this article are included in the article's Creative Commons license, unless indicated otherwise in a credit line to the material. If material is not included in the article's Creative Commons license and your intended use is not permitted by statutory regulation or exceeds the permitted use, you will need to obtain permission directly from the copyright holder. To view a copy of this license, visit http://creativecommons.org/ licenses/by/4.0/

(C) The Author(s) 2020 